\title{
Heavy-ion fusion hindrance in systems with positive $Q$ value
}

\author{
C.L. Jiang, ${ }^{\text {a }}$ \\ Physics Division, Argonne National Laboratory, Argonne, IL 60439, USA
}

\begin{abstract}
The detailed behavior of the newly observed heavy-ion fusion hindrance for systems with positive $Q$ value is not yet known well. Does an $S$ factor maximum occur also for these systems at very low energies? This is still an open question and is discussed in this presentation of the experimental activities.
\end{abstract}

\section{Introduction}

About 10 years ago a falloff of the fusion cross sections at extreme sub-barrier energies was discovered, which was steeper than theoretical predictions [1]. This effect is now known as fusion hindrance and is believed to de due to the saturation property of nuclear matter [2]. It was first found for medium-mass systems which all have negative fusion $Q$ values, where a maximum in the $S$ factor is required due to the energy conservation. Detailed systematics of fusion hindrance has been established and nuclear structure effects have been investigated [1,3].

Fusion reactions between lighter heavy-ions are very important in explosive stellar burning processes. Reactions such as ${ }^{12} \mathrm{C}+{ }^{12} \mathrm{C},{ }^{12} \mathrm{C}+{ }^{16} \mathrm{O}$ and ${ }^{16} \mathrm{O}+{ }^{16} \mathrm{O}$ are the main processes during the carbon and oxygen burning stages. Furthermore, other fusion reactions, e.g. ${ }^{24} \mathrm{O}+{ }^{24} \mathrm{O},{ }^{28} \mathrm{Ne}$ $+{ }^{28} \mathrm{Ne}$ and ${ }^{34} \mathrm{Ne}+{ }^{34} \mathrm{Ne}$ are involved in the evolution of the inner crust of accreting neutron stars [4]. For these lighter systems it was found that the fusion cross sections also fall off more steeply than predicted by previous extrapolations and theoretical (e.g. potential penetration) calculations [5], leading to a large decrease of the reaction rates at astrophysical energies [6]. This phenomenon displays also the hindrance behavior related to the saturation property of nuclear matter. Since the fusion $Q$ values of these lighter heavy-ion reactions are always positive, an $S$ factor maximum is not required. The detailed behavior of the hindrance and whether an $S$ factor maximum also appears at very low energies thus become an interesting subject. Previously the hindrance was quantitatively described by a phenomenological fit to the logarithmic derivative with the formula [5]:

$$
L(E)=A_{0}+B_{0} / E^{N_{p}},
$$

here $N_{p}$ was taken as 1.5. An $S(E)$ plot for the reaction ${ }^{12} \mathrm{C}$ $+{ }^{12} \mathrm{C}$ together with different extrapolations is shown in Fig. 1 [7-10]. Here, extrapolations of hindrance prescription with various options of $N_{p}=1-1.5$ are included [11]. Obviously, there are big uncertainties of "expectations" in

\footnotetext{
a e-mail: jiang@phy.anl.gov
}

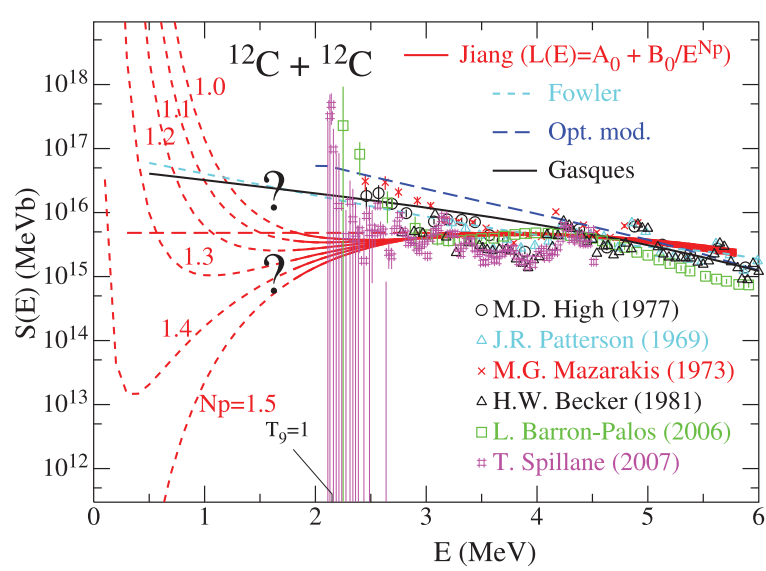

Fig. 1. $S(E)$ for fusion ${ }^{12} \mathrm{C}+{ }^{12} \mathrm{C}$. Six extrapolated $S(E)$ (red curves) are obtained with different $N_{p}=1.0-1.5$.

the energy range of astrophysical interest. The final answer must be obtained from the experimental measurements.

Two kinds of experiments have been performed recently in order to study the fusion hindrance in systems with positive $Q$ values : measurements for reactions of intermediatemass region and reactions of ${ }^{12} \mathrm{C}+{ }^{13} \mathrm{C}$ and ${ }^{12} \mathrm{C}+{ }^{12} \mathrm{C}$.

\section{Intermediate-mass systems with positive $Q$ values}

Due to the difficulties in the direct measurements for these lighter systems an extension of measurements of fusion excitation functions towards intermediate-mass systems with positive $Q$ values is a natural step for these studies. The four reactions ${ }^{28} \mathrm{Si}+{ }^{30} \mathrm{Si},{ }^{27} \mathrm{Al}+{ }^{45} \mathrm{Sc},{ }^{36} \mathrm{~S}+{ }^{48} \mathrm{Ca}$ and ${ }^{40} \mathrm{Ca}+{ }^{48} \mathrm{Ca}$ with fusion $Q$ values of $14.3,9.63,7.55$ and $4.56 \mathrm{MeV}$, respectively, have been measured down to the $\mu b$ or sub- $\mu b$ region at ANL and INFN, Legnaro, [12,13].

Three excitation functions for fusion reactions with positive $\left({ }^{28} \mathrm{Si}+{ }^{30} \mathrm{Si}\right.$ and ${ }^{27} \mathrm{Al}+{ }^{45} \mathrm{Sc}$ [12]) or slightly negative

This is an Open Access article distributed under the terms of the Creative Commons Attribution-Noncommercial License 3.0, which permits unrestricted use, distribution, and reproduction in any noncommercial medium, provided the original work is properly cited. 


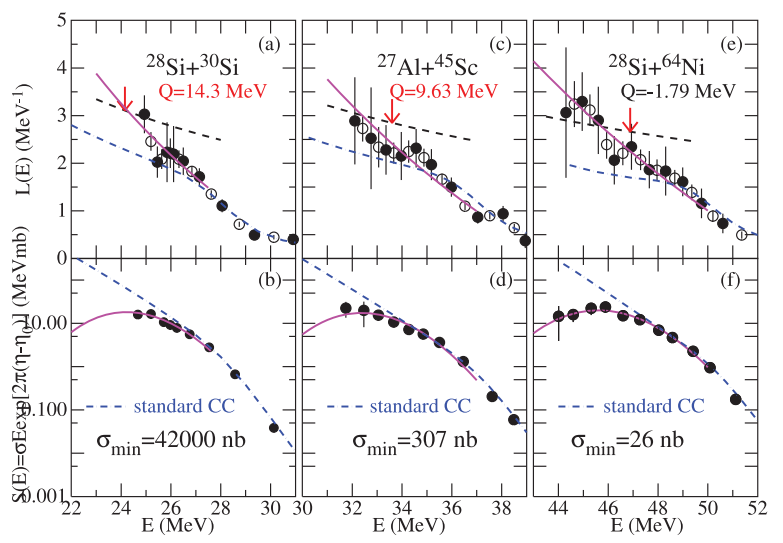

Fig. 2. $S(E)$ and $L(E)$ for the systems ${ }^{28} \mathrm{Si}+{ }^{30} \mathrm{Si},{ }^{27} \mathrm{Al}+{ }^{45} \mathrm{Sc}$ and ${ }^{28} \mathrm{Si}+{ }^{64} \mathrm{Ni}$.

$\left({ }^{28} \mathrm{Si}+{ }^{64} \mathrm{Ni}[1]\right) Q$ value, are compared in Fig. 2. The lowest cross sections $\left(\sigma_{\min }\right)$ measured in these experiments are given in the figure. The behavior of these three systems for both $L(E)$ and $S(E)$ is very similar. At the lowest energies, standard CC calculations (shown by the blue dasheddotted curves) overpredict the cross sections. An $S$ factor maximum is observed only for the system ${ }^{28} \mathrm{Si}+{ }^{64} \mathrm{Ni}$, where the $Q$ value is slightly negative, but the cross sections have been measured down to $26 \mathrm{nb}$. For the other two systems, ${ }^{28} \mathrm{Si}+{ }^{30} \mathrm{Si}$ and ${ }^{27} \mathrm{Al}+{ }^{45} \mathrm{Sc}$, the measurements need to be extended to lower energies before a similar conclusion can be reached (the backgrounds were too high, which prevented us from expanding the measurements to lower energies).

Another series of measurements are show in Fig. 3, where results for the system ${ }^{36} \mathrm{~S}+{ }^{48} \mathrm{Ca}(Q=7.55 \mathrm{MeV})$ are compared with measurements in the system ${ }^{48} \mathrm{Ca}+$ ${ }^{48} \mathrm{Ca}(Q=-2.99 \mathrm{MeV})$ [13]. The lowest cross sections measured in these experiments are also listed in the figures. The red arrows in Fig. 3 (and also in Fig. 2) give the predicted energy locations of the $S$ factor maximum, obtained from the hindrance systematics (see Ref. [5], which surveyed the fusion excitation functions of 'stiff' systems from medium-mass nuclei down to lighter systems around $\left.{ }^{16} \mathrm{O}+{ }^{16} \mathrm{O}\right)$. The systematics is expressed by the following equations :

$$
L_{s}^{e m p}=2.33+580 / \zeta\left(\mathrm{MeV}^{-1}\right),
$$

and

$$
E_{s}^{e m p}=\left(0.495 \zeta / L_{s}^{e m p}(\zeta)\right)^{2 / 3}(\mathrm{MeV}) .
$$

The results of $S(E)$ and $L(E)$ for the systems ${ }^{36} \mathrm{~S}+$ ${ }^{48} \mathrm{Ca}$ and ${ }^{48} \mathrm{Ca}+{ }^{48} \mathrm{Ca}$ are very similar, though there is a big difference between their fusion $Q$ values : 7.55 and $-2.99 \mathrm{MeV}$, respectively [13]. An exponential increase of $S(E)$ with decreasing energy is followed by a change in slope at the lowest energies, where the $S(E)$ falls below the standard CC predictions (blue dash-dotted curves). That is, hindrance behaviors are observed in these systems as well as for those shown in Fig. 2. It seems however, that the detailed patterns of these $S(E)$ and $L(E)$ curves are different

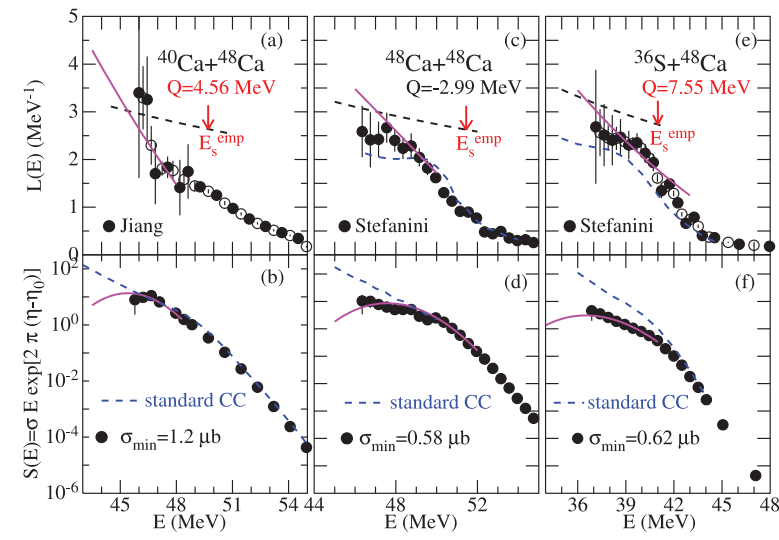

Fig. 3. $S(E)$ and $L(E)$ for the systems ${ }^{40} \mathrm{Ca}+{ }^{48} \mathrm{Ca},{ }^{48} \mathrm{Ca}+{ }^{48} \mathrm{Ca}$ and ${ }^{36} \mathrm{~S}+{ }^{48} \mathrm{Ca}$.

from those observed in Fig. 2. For these two systems, no $S$ factor maximum has been observed in the measured energy regions (which extend much lower than the predicted values of the $S$ factor maximum $E_{s}^{e m p}$ ). This was unexpected, because both ${ }^{48} \mathrm{Ca}$ and ${ }^{36} \mathrm{~S}$ are closed shell nuclei. Nevertheless, since the $Q$ value of ${ }^{48} \mathrm{Ca}+{ }^{48} \mathrm{Ca}$ is negative, there should be an $S$ factor maximum. It should be mentioned that the energy locations of $E_{s}^{e m p}$ have to be considered as upper limits, especially for systems which do not involve 'stiff' nuclei. One possibility could be that the higher $N-Z$ values for the systems ${ }^{48} \mathrm{Ca}+{ }^{48} \mathrm{Ca}$ and ${ }^{36} \mathrm{~S}+{ }^{48} \mathrm{Ca}$ push the fusion hindrance to lower energies (as seen in open systems in the medium mass region [1]).

This consideration encouraged us to again measure the reaction ${ }^{40} \mathrm{Ca}+{ }^{48} \mathrm{Ca}$, which is a positive $Q$ value system, and the $N-Z$ value is eight, smaller than the $N-Z$ of the other two systems discussed above, 16 and 12 , respectively.

This was a cooperative study of ANL and LNL, measured at LNL. The measurement has extended downward by two orders of magnitude with respect to previous cross section data $[14,15]$. The results are shown in Fig. 3a and $3 \mathrm{~b}$ [16]. One can see that the measured $L(E)$ crosses the constant $S$ factor curve at the lowest energies. The crossing point, at $E_{s}=45.4 \mathrm{MeV}$, corresponds to the $S$ factor maximum which can be recognized in Fig. 3b. Evidently, more data points at even lower energies are desirable. This result is a first indication of an $S$ factor maximum observed in a system with a positive $Q$ value. If confirmed in other systems with positive $Q$ values, this effect will play an important role in nuclear astrophysics.

The correlation between fusion hindrance and the neutron excess $N-Z$ observed in the two systems of $\mathrm{Ca}+\mathrm{Ca}$ pushed us to study further another member, ${ }^{40} \mathrm{Ca}+{ }^{40} \mathrm{Ca}$, which has $N-Z=0$ and was measured previously only to $0.22 \mathrm{mb}$, well above the hindrance region.

Results of the system ${ }^{40} \mathrm{Ca}+{ }^{40} \mathrm{Ca}$ are shown in Fig. $4 \mathrm{a}$ and $4 \mathrm{~b}$ [17]. There may be an $S$ factor maximum nearby the predicted energy (by Eqs. (2) and (3)) as indicated by a red arrow in Fig. 4a. For three systems of $\mathrm{Ca}+\mathrm{Ca}$, with $N-Z=0,8$ and 16 , the fact that the measured values of 


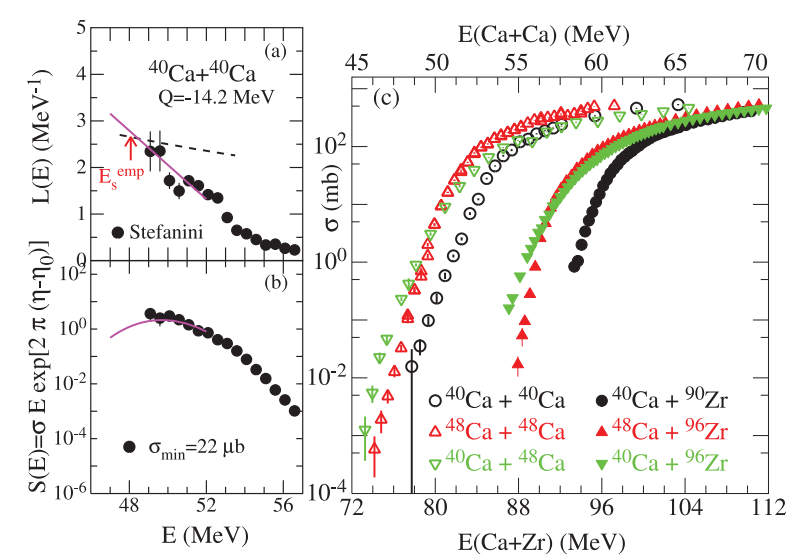

Fig. 4. (a) and (b): $L(E)$ and $S(E)$ for reaction ${ }^{40} \mathrm{Ca}+{ }^{40} \mathrm{Ca}$. (c) excitation functions for $\mathrm{Ca}+\mathrm{Ca}$ and $\mathrm{Ca}+\mathrm{Zr}$.

$E_{s}^{e m p}-E_{s}=\sim 0,4$ and $>7 \mathrm{MeV}$, respectively, demonstrates an interesting nuclear structure effect. Surprisingly, fusion reactions with nucleus ${ }^{48} \mathrm{Ca}$ behaves ${ }^{64} \mathrm{Ni}$ or ${ }^{96} \mathrm{Zr}$ but not as ${ }^{58} \mathrm{Ni}$ or ${ }^{90} \mathrm{Zr}$, though it is a double magic nucleus in nuclear structure aspect. In addition, the nucleus ${ }^{36} \mathrm{~S}$ behaves as ${ }^{48} \mathrm{Ca}$ in the fusion reaction though it is a singly magic nucleus. It will be very interesting to measure more systems of $\mathrm{S}+\mathrm{Ca}$ down to the hindrance region to further study this observation.

There is another important point which should be explored. It was mentioned in Ref. [16] that the changing of the excitation functions from ${ }^{40} \mathrm{Ca}+{ }^{40} \mathrm{Ca}$ to ${ }^{40} \mathrm{Ca}+{ }^{48} \mathrm{Ca}$, then to ${ }^{40} \mathrm{Ca}+{ }^{48} \mathrm{Ca}$, is very similar with the change from ${ }^{40} \mathrm{Ca}+{ }^{90} \mathrm{Zr}$ to ${ }^{48} \mathrm{Ca}+{ }^{96} \mathrm{Zr}$, then to ${ }^{40} \mathrm{Ca}+{ }^{96} \mathrm{Zr}$. The comparisons are shown in Fig. 4c.

The nucleus ${ }^{40} \mathrm{Ca}$ is a doubly closed shell nuclei, and the system ${ }^{40} \mathrm{Ca}+{ }^{40} \mathrm{Ca}$ is a stiff one. Cross sections of ${ }^{48} \mathrm{Ca}$ $+{ }^{48} \mathrm{Ca}$ are higher than those of ${ }^{40} \mathrm{Ca}+{ }^{40} \mathrm{Ca}$ due to more coupling effects (many 'valence' neutrons) in the coupledchannels (CC) calculations, the larger radius in ${ }^{48} \mathrm{Ca}$ is responsible for that. Cross sections of ${ }^{40} \mathrm{Ca}+{ }^{48} \mathrm{Ca}$ are even higher than of ${ }^{48} \mathrm{Ca}+{ }^{48} \mathrm{Ca}$ at low energies, due to the contribution of transfer reactions. The $Q$ value of transfer reactions are more positive in the non-symmetrical system ${ }^{40} \mathrm{Ca}+{ }^{48} \mathrm{Ca}$. Interestingly, the changing tendency mentioned above, is always true, not only for $\mathrm{Ca}+\mathrm{Ca}$, but also for many other combinations, e.g. for $\mathrm{Ca}+\mathrm{Zr}$ in Fig. $4 \mathrm{c}$ and $\mathrm{Ni}+\mathrm{Ni}, \mathrm{S}+\mathrm{Ni}, \mathrm{S}+\mathrm{Zr}$ and $\mathrm{Si}+\mathrm{Ni}$ in Fig. 5a and $5 \mathrm{~b}$, etc. We have found the same changing tendency even in the lighter mass systems, which will be discussed in the following section.

\section{System ${ }^{12} \mathrm{C}+{ }^{13} \mathrm{C}$ and ${ }^{12} \mathrm{C}+{ }^{12} \mathrm{C}$}

The reaction ${ }^{12} \mathrm{C}+{ }^{13} \mathrm{C}$ has been measured down to $\sim 3$ $\mathrm{MeV}$ by Dayras [18]. The excitation function is smoother than in ${ }^{12} \mathrm{C}+{ }^{12} \mathrm{C}$ where there are many resonances in the excitation function. There was a flat region $(E=3-4.5$ $\mathrm{MeV}$ ) on Dayras' $S(E)$ curve (Fig. 6). Because the extrapolation from Fowler's [7] is going up, and the expectation

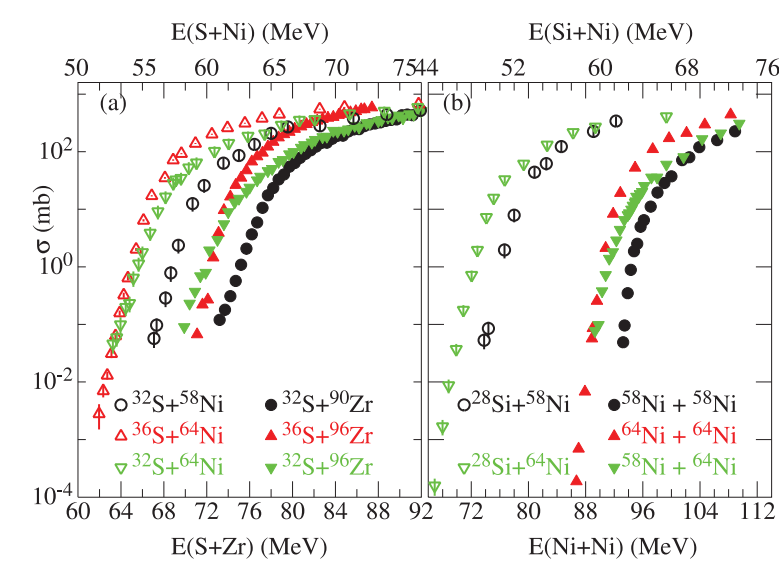

Fig. 5. Excitation functions for systems $\mathrm{S}+\mathrm{Ni}, \mathrm{S}+\mathrm{Zr}, \mathrm{Ni}+\mathrm{Ni}$ and $\mathrm{Si}+\mathrm{Ni}$.

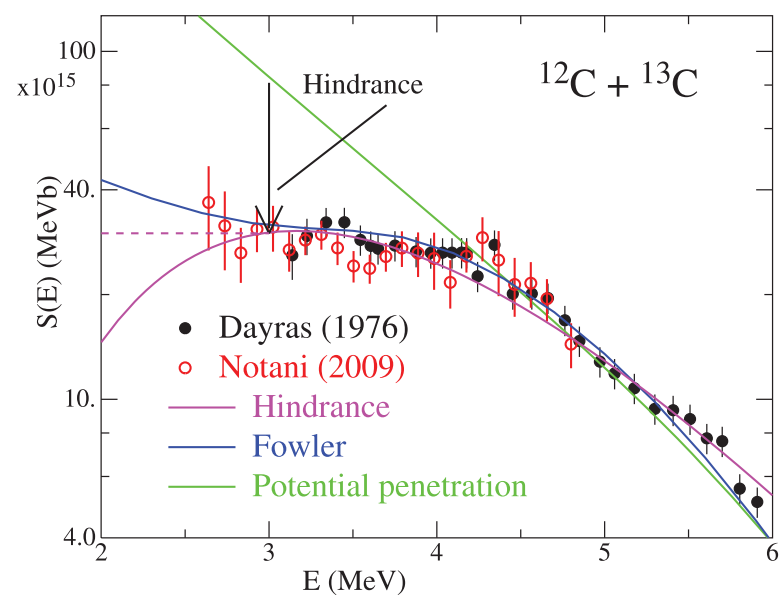

Fig. 6. The $S(E)$ for systems ${ }^{12} \mathrm{C}+{ }^{13} \mathrm{C}$.

from the hindrance model is going down when the energy decreases, with an extension of the measurement towards lower energies one may be able to determine which model works better.

An experiment was performed recently by measuring the $\beta$-decay of ${ }^{24} \mathrm{Na}$ from the reaction ${ }^{12} \mathrm{C}+{ }^{13} \mathrm{C}$ in the energy range of $2.5-5 \mathrm{MeV}$ [19]. The experimental results (Fig. 6) show a continuously flat $S$ factor to the lowest measured energy, $\sim 2.5 \mathrm{MeV}$ (the lowest cross section measured is about $4 \mathrm{nb}$ ). Now we know that the valence neutron in ${ }^{13} \mathrm{C}$ introduces more coupling and pushes the hindrance towards lower energies. It is similar to the case where the extra neutrons of ${ }^{40} \mathrm{Ca}+{ }^{48} \mathrm{Ca}$ push the hindrance towards lower energies than in the reaction ${ }^{40} \mathrm{Ca}+{ }^{40} \mathrm{Ca}$.

Excitation functions of ${ }^{12} \mathrm{C}+{ }^{12} \mathrm{C},{ }^{13} \mathrm{C}+{ }^{13} \mathrm{C}$ and ${ }^{12} \mathrm{C}$ $+{ }^{13} \mathrm{C}$ are compared in Fig. 7. We can recognize (though not very easily) that the fusion cross sections of ${ }^{13} \mathrm{C}+{ }^{13} \mathrm{C}$ are higher than those of ${ }^{12} \mathrm{C}+{ }^{12} \mathrm{C}$, and the ones of ${ }^{12} \mathrm{C}+$ ${ }^{13} \mathrm{C}$ are higher than those of ${ }^{13} \mathrm{C}+{ }^{13} \mathrm{C}$. This phenomenon is also true at higher energies and lower energies as shown in Fig. $8 \mathrm{~b}$ and $8 \mathrm{a}$, respectively.

In order to suppress the strong influence from the penetration through the Coulomb potential, Fowler used the 


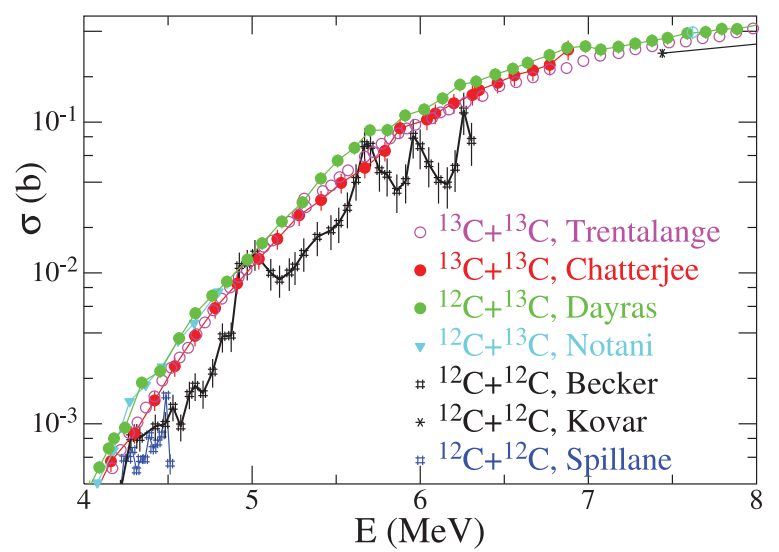

Fig. 7. Excitation functions for systems ${ }^{13} \mathrm{C}+{ }^{13} \mathrm{C},{ }^{12} \mathrm{C}+{ }^{13} \mathrm{C}$ and ${ }^{12} \mathrm{C}+{ }^{12} \mathrm{C}$ at $4-8 \mathrm{MeV}$.

nuclear factor representation [7]:

$$
\text { nuclear }- \text { factor }=\sigma E \exp (2 \pi \eta+g E) .
$$

For ${ }^{12} \mathrm{C}+{ }^{12} \mathrm{C}$, it is

$$
\text { nuclear }- \text { factor }=\sigma E \exp (87.26 / \sqrt{E}+0.46 E) .
$$

The results of applying Eq. (5) to ${ }^{12} \mathrm{C}+{ }^{13} \mathrm{C},{ }^{13} \mathrm{C}+$ ${ }^{13} \mathrm{C}$ and ${ }^{12} \mathrm{C}+{ }^{12} \mathrm{C}$ are shown in Fig. 9, which illustrates the ratio between the different curves in a linear scale. By ignoring the data from Spillane (blue symbols) at first, one can see two trends more evidently in Fig. 9: 1) Similar to the discussions for $\mathrm{Ca}+\mathrm{Ca}$, cross sections of ${ }^{13} \mathrm{C}+{ }^{13} \mathrm{C}$ are higher than those of ${ }^{12} \mathrm{C}+{ }^{12} \mathrm{C}$, and cross sections of ${ }^{12} \mathrm{C}$ $+{ }^{13} \mathrm{C}$ are higher than those of ${ }^{13} \mathrm{C}+{ }^{13} \mathrm{C}$. The $Q$ values of transfer reactions in ${ }^{12} \mathrm{C}+{ }^{13} \mathrm{C}$ are also higher than for the two symmetric systems. It should be noted, only one neutron of ${ }^{13} \mathrm{C}$ is outside the ${ }^{12} \mathrm{C}$ core, while eight neutrons of ${ }^{48} \mathrm{Ca}$ are outside the ${ }^{40} \mathrm{Ca}$ core; at the same time, the cross sections of reaction ${ }^{12} \mathrm{C}+{ }^{13} \mathrm{C}$ are larger than the ones in ${ }^{12} \mathrm{C}+{ }^{12} \mathrm{C}$ by only a factor of about 2 or 3 , while in the case of $\mathrm{Ca}+\mathrm{Ca}$, the difference could be as much as several orders of magnitude. 2) There are many resonances in the ${ }^{12} \mathrm{C}+{ }^{12} \mathrm{C}$ curve, but the resonance peaks are never higher than the curve of ${ }^{12} \mathrm{C}+{ }^{13} \mathrm{C}[20]$.

Imanishi explained these molecular resonances in the reaction ${ }^{12} \mathrm{C}+{ }^{12} \mathrm{C}$ with a virtual excitation of the 4.44 $\mathrm{MeV}$ state of ${ }^{12} \mathrm{C}$ coinciding with an isolated eigenstate of ${ }^{24} \mathrm{Mg}$ [21]. In the case of ${ }^{12} \mathrm{C}+{ }^{13} \mathrm{C}$, with the couplings to the extra neutron, a virtual excitation of the $3.684 \mathrm{MeV}$ state of ${ }^{13} \mathrm{C}$ can often coincide with one of the many overlapping eigenstates of ${ }^{25} \mathrm{Mg}$, resulting in 'resonances' occurring continuously along the whole excitation function of ${ }^{12} \mathrm{C}+{ }^{13} \mathrm{C}$. The coupling to the extra neutron supplies fully the strength of the molecular resonances. Thus, one may understand that why, the excitation function of ${ }^{12} \mathrm{C}+$ ${ }^{13} \mathrm{C}$ is the upper limit of the excitation function of ${ }^{12} \mathrm{C}+$ ${ }^{12} \mathrm{C}$, including the strong resonances.

Spillane et al., found a very strong resonance at about 2.1 MeV in ${ }^{12} \mathrm{C}+{ }^{12} \mathrm{C}[10]$, whereas Cooper et al., suggested that there might be a huge resonance at about 1.5

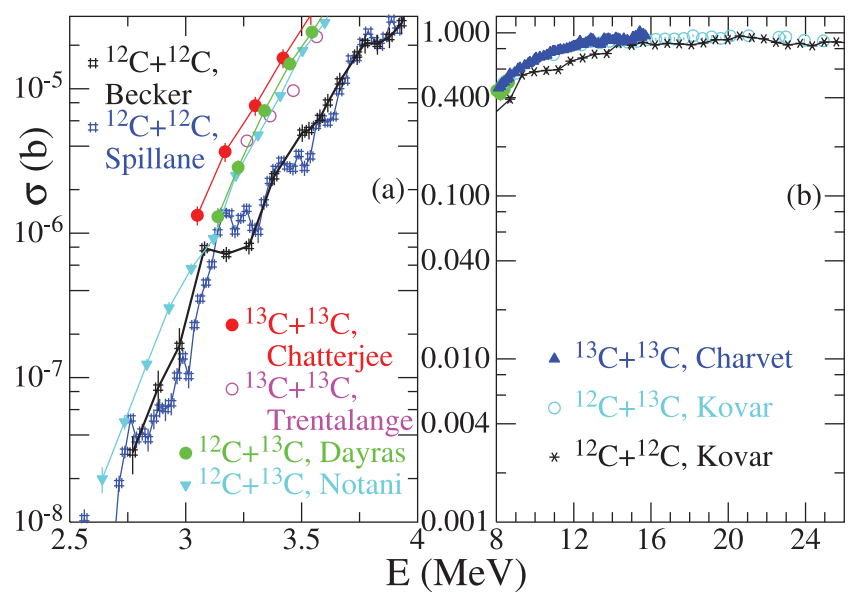

Fig. 8. Excitation functions for systems ${ }^{13} \mathrm{C}+{ }^{13} \mathrm{C},{ }^{12} \mathrm{C}+{ }^{13} \mathrm{C}$ and ${ }^{12} \mathrm{C}+{ }^{12} \mathrm{C}$ at $2.5-4$ and $8-26 \mathrm{MeV}$, respectively.

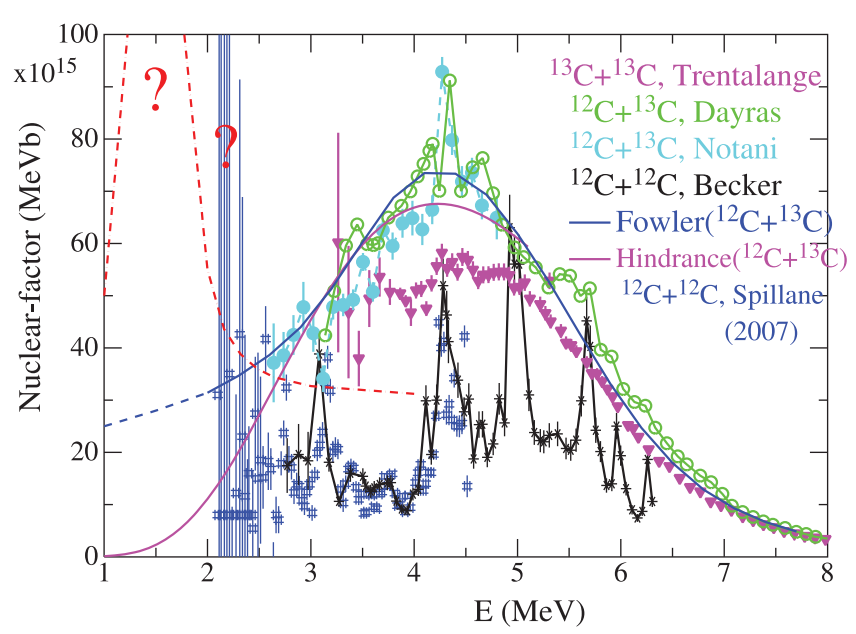

Fig. 9. Nuclear factors for systems ${ }^{13} \mathrm{C}+{ }^{13} \mathrm{C},{ }^{12} \mathrm{C}+{ }^{13} \mathrm{C}$ and ${ }^{12} \mathrm{C}$ $+{ }^{12} \mathrm{C}$ atlow energies.

$\mathrm{MeV}$ (orange dash-dotted curve in Fig. 9) in order to explain the ignition of the carbon burning in the superburst [22]. Because these two resonances are much higher than the two curves, given by Fowler [7] and the hindrance prescription, which can be looked at as the upper and lower limits for the data of ${ }^{12} \mathrm{C}+{ }^{13} \mathrm{C}$ (see Fig. 6), one may argue that these two resonances are questionable. Recently, Zickerfoose et al., remeasured the excitation function of ${ }^{12} \mathrm{C}+$ ${ }^{12} \mathrm{C}$ with the charged particle method [23]. They did not see the resonance found by Spillane.

\section{A new technique for measuring ${ }^{12} \mathrm{C}+{ }^{12} \mathrm{C}$ at low energies}

The most important exit channels of the system ${ }^{12} \mathrm{C}+{ }^{12} \mathrm{C}$ are ${ }^{23} \mathrm{Na}+\mathrm{p}$ and ${ }^{20} \mathrm{Ne}+\alpha$, with discrete $Q$-value spectra. Two different techniques have been used in the past to 
measure the low energy excitation function of ${ }^{12} \mathrm{C}+{ }^{12} \mathrm{C}$, detecting either charged particles or $\gamma$-rays. Several experiments measured $\alpha$ 's and protons with Si-detectors [8]. The ubiquitous hydrogen contamination in the target (H and D) gave elastically scattered protons and deutrons in the low energy part and reaction particles $\mathrm{D}\left({ }^{12} \mathrm{C}, \mathrm{p}\right)$ in the high energy part of the charged particle energy spectra, restricting the measurements from going to lower beam energies.

The $\gamma$-ray detection, with large volume HPGe or Ge(Li) detectors, has been the main method used to measure this system in recent years $[9,10]$. Most of these experiments only measured the $\gamma$-rays emitted from the first excited states in ${ }^{23} \mathrm{Na}(0.440 \mathrm{MeV})$ and in ${ }^{20} \mathrm{Ne}(1.634 \mathrm{MeV})$. The excited states reached via $\alpha$-emission cascade nearly $100 \%$ through the first excited state of ${ }^{20} \mathrm{Ne}$, while the excited states reached via proton-emission cascade $\sim 65 \%$ through the first excited state of ${ }^{23} \mathrm{Na}$. The population corrections for the ground states were taken from measurements of light charged particles. At low beam energies the $\gamma$-ray spectra suffered from intense backgrounds at $E_{\gamma}=2.36$ $\mathrm{MeV}$ (from the reaction $\mathrm{H}\left({ }^{12} \mathrm{C}, \gamma\right){ }^{13} \mathrm{~N}$ ) and at $E_{\gamma}=3.09$ $\mathrm{MeV}$ (from the reaction $\mathrm{D}\left({ }^{12} \mathrm{C}, \mathrm{p} \gamma\right){ }^{13} \mathrm{C}$ ).

All these excitation functions have been measured in 'singles' mode. No coincidence studies have been reported in the literature. Small amounts of contamination of elements lighter than carbon in the target would introduce serious background problems in the singles mode, especially at very low energies. Examples are the two resonances found at about 2.1 and $1.5 \mathrm{MeV}$, but recently it was reported that these were due to the contaminations in the target [23].

In order to avoid these background issues we have developed a particle- $\gamma$ coincidence technique to measure this reaction, which, however, requires more efficient detection of the reaction products. There are advantages to use Gammasphere (at ANL) to detect the most important $\gamma$-rays, $E_{\gamma}=1.63$ and $0.440 \mathrm{MeV}$, since the efficiency for these two lines will be relatively high $(\geq 10 \%)$. The background spectrum will be significant in singles, especially at low beam energies where the ${ }^{12} \mathrm{C}+{ }^{12} \mathrm{C}$ fusion cross section is very small. Measurements with coincidences can suppress many $\gamma$-ray background processes. The coincidence with a light particle (proton and $\alpha$ ) can be very efficient, since modern, Si-array detectors can be arranged with rather large solid angles.

In a recent experiment [28], we used one double-sided stripped-Si detector, DSSD, to measure the $\alpha^{\prime} s$ and protons in conjunction with Gammasphere. There are 16 rings in the DSSD, corresponding to different angular range with a solid angle coverage about $7.4 \%$ of $4 \pi$. The DSSD was put either in the forward angles, $21.7-38.6^{\circ}$, or the backward position, $141.4-158.3^{\circ}$, respectively. Thin carbon targets, about $40 \mu \mathrm{m} / \mathrm{cm}^{2}$ (either isotopic or natural) were used.

A two dimensional coincidence spectrum $\left(E_{\gamma}\right.$ versus $E_{\text {particle }}$ ) at $E_{\text {lab }}=10 \mathrm{MeV}$ and around $30^{\circ}$ (the sum of three rings) is shown in Fig. 10. Some groups from the reactions ${ }^{12} \mathrm{C}\left({ }^{12} \mathrm{C}, p\right)$ and ${ }^{12} \mathrm{C}\left({ }^{12} \mathrm{C}, \alpha\right)$, in coincidences with the $\gamma$-rays of 440 and $1634 \mathrm{keV}$, respectively, are indicated in the figure.

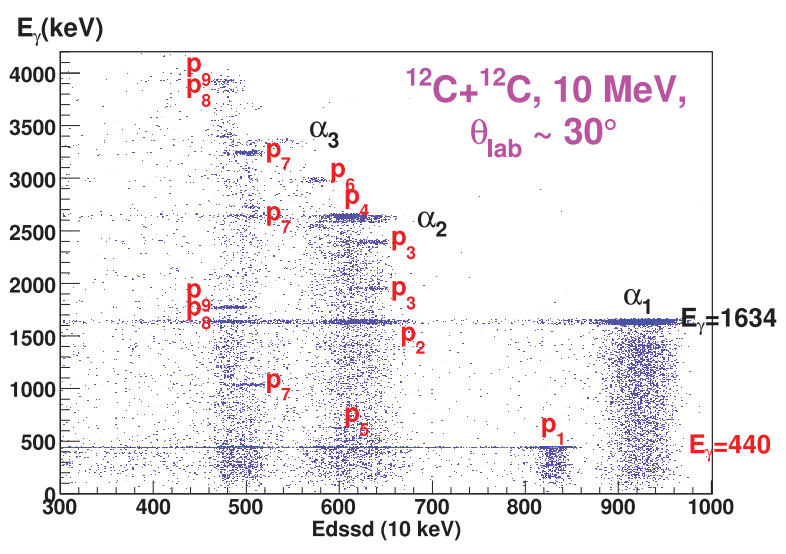

Fig. 10. A particle- $\gamma$ coincidence spectrum from the measurement of the fusion reaction ${ }^{12} \mathrm{C}+{ }^{12} \mathrm{C}$ at $E_{c m}=5 \mathrm{MeV}$ and $\theta_{\text {particle }} \sim 30^{\circ}$. The particle groups coincide with the $\gamma$-lines at 1634 (black), 440 (red) keV and others are indicated.

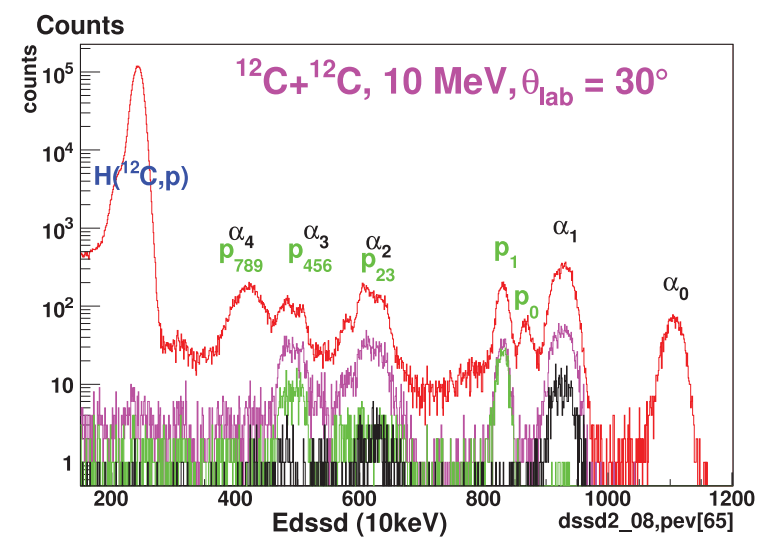

Fig. 11. Particle energy spectra detected from the DSSD. Red: without coincidence, magenta: coincidence with $\gamma$-ray from any one of the $110 \mathrm{Ge}$-detectors in the Gammasphere, green or black, coincidence with a $\gamma$-ray of $E_{\gamma}=440$ or $1634 \mathrm{keV}$, respectively.

A particle spectra from ring- 8 of the $\operatorname{DSSD}\left(\theta_{\text {lab }}=30^{\circ}\right.$ at $E_{\text {lab }}\left({ }^{12} \mathrm{C}\right)=10 \mathrm{MeV}$ is shown in Fig. 11. The red spectrum was obtained without a coincidence and suffered very much by the products of the reaction $\mathrm{H}\left({ }^{12} \mathrm{C}, \mathrm{p}\right)$. A corresponding spectrum (magenta) is shown for events which are in coincidence with a $\gamma$-ray from any one of the 110 Ge-detectors of the Gammasphere. The spectra in black and green are particle groups of proton and $\alpha$, which coincide with a $\gamma$-ray of $E_{\gamma}=440$ or $1634 \mathrm{keV}$, respectively. The $\mathrm{H}\left({ }^{12} \mathrm{C}, \mathrm{p}\right)$ contaminating reaction, does not produce coincidences, and under the peaks around $3-4 \mathrm{MeV}$, there are contributions from other backgrounds, like the reaction $\mathrm{D}\left({ }^{12} \mathrm{C}, \mathrm{d}\right)$, which does not have coincidences either.

From these figures one may recognize the superiority of discriminating background by the coincidence method. 


\section{EPJ Web of Conferences}

In fact, these are the first coincidence spectra which can be found in the literature for the study of the fusion reaction ${ }^{12} \mathrm{C}+{ }^{12} \mathrm{C}$

From the results of this experiment we conclude that if several DSSD's are installed, and with a beam current of $200 \mathrm{p} \mu \mathrm{A}$, at about $E=2 \mathrm{MeV}$ (with expected cross section of about $10 \mathrm{pb}), 200$ good coincidence events can be accumulated during a long run of about 10 days. It will not be easy, but not impossible.

This technology can also be used in the measurements of many other fusion systems of lighter heavy-ions in an efficient way.

\section{Summary}

A first evidence of an $S$ factor maximum has been observed in a system with a positive $Q$ value, ${ }^{40} \mathrm{C}+{ }^{48} \mathrm{C}$. If confirmed in other systems with positive $Q$ values, this effect will play an important role in nuclear astrophysics.

A nuclear structure effect, the influence of the neutron excess $N-Z$ on the energy of the $S$ factor maximum, $E_{s}$ has been found in the intermediate mass region.

The excitation function of ${ }^{12} \mathrm{C}+{ }^{13} \mathrm{C}$ can be viewed as the upper limit of the excitation function of ${ }^{12} \mathrm{C}+{ }^{12} \mathrm{C}$ including the strong resonances. It seems that the two resonances observed or suggested previously at 2.1 and 1.5 $\mathrm{MeV}$, are questionable.

A similarity between the changing tendency of the excitation functions for light mass system, $\mathrm{C}+\mathrm{C}$, and heavier mass system, e.g. $\mathrm{Ca}+\mathrm{Ca}, \mathrm{Ni}+\mathrm{Ni}$ etc., has been observed. The physics of that phenomenon needs to be explored.

A new technique using particle- $\gamma$ coincidence has been developed for the measurement of very low cross sections. It is expected that the measurement of reaction ${ }^{12} \mathrm{C}+{ }^{12} \mathrm{C}$ can be pushed down to the level of $\sim 10 \mathrm{pb}$ by using this technique.

\section{Acknowledgments}

This work was supported by the US Department of Energy, Office of Nuclear Physics, under Contract No. DE-AC0206CH11357.

\section{References}

1. C.L. Jiang et al., Phys. Rev. Lett. 89, 052701 (2002); 93, 012701 (2004); Phys. Rev. C 71, 044613 (2005); Phys. Lett. B 640, 18 (2006).

2. Ş. Mişicu et al., Phys. Rev. Lett. 96,112701 (2006).

3. C.L. Jiang et al., Phys. Rev. C 69, 014604 (2004); C 73, 014613 (2006); C 75, 057604 (2007).

4. P. Haenel et al., Astron. Astrophys. 229, 117 (1990); 404, L33 (2003); C.J. Horowitz et al., Phys. Rev. E 79, 066101 (2007).

5. C.L. Jiang et al., Phys. Rev. C 75, 015803 (2007); C 79, 044601 (2009).
6. L.R. Gasques et al., Phys. Rev. C 76, 035802 (2007).

7. W. Fowler et al., Annu. Rev. Astrophy 13, 69 (1975).

8. L.J. Patterson et al., As. J. 157, 367 (1969); M. Mazarakis et al., Phys. Rev. C 7, 1280 (1973); H.W. Becker et al., Phys. Rev. Lett. 38, 337 (1977).

9. M.D. High et al., Nucl. Phys. A 282, 182 (1977); K.U. Kettner et al., Z. Phys. A 298, 65 (1980); L.R. BarronPalos et al., Nucl. Phys. A 779, 218 (2006);

10. T. Spillane et al., Phys. Rev. Lett. 98, 122501 (2007).

11. C.L. Jiang et al., Proceedings of International Nuclear Physics Conference, July 4-9, 2010, Vancouver, Canada.

12. C.L. Jiang et al., Phys. Rev. C 78, 017601 (2008); C 81, 024611 (2010).

13. A.M. Stefanini et al., Phys. Rev. C 78044607 (2008); Phys. Lett. B 679, 95 (2009).

14. H.A. Aljuwair et al., Phys. Rev. C 30, 1223 (1984).

15. M. Trotta et al., Phys. Rev. C 65, 011601R (2001).

16. C.L. Jiang et al., Phys. Rev. C 82, 041601 (2010).

17. A.M. Stefanini et al., in preparation for Phys. Rev. C, (2011).

18. R.A. Dayras et al., Nucl. Phys. A 265, 153 (1975).

19. M. Notani et al., Nucl. Phys. A 834, 192C (2010).

20. X. Tang et al., in preparation for Phys. Rev. Lett. (2011).

21. B. Imanishi et al., Phys. Lett. B 27, 267 (1968); Phys. Rev. C 35, 359 (1987).

22. R.L. Cooper et al., Astrophys J. 702, 660 (2009).

23. J. Zickerfoose, Thesis, U. Conn. (2011). (2009).

24. D.G. Kovar et al., Phys. Rev. C 20, 1305 (1979);

25. S. Trentalange et al., Nucl. Phys. A 483, 406 (1988);

26. M. Chatterjee et al., Nucl. Phys. A 333, 273 (1980);

27. J.L. Charvet et al., Nucl. Phys. A 376, 292 (1982);

28. X. Fang et al., in preparation for Nucl. Instr. and Meth. A, (2011). 